\title{
Brain structural alterations in MOG antibody diseases: a comparative study with AQP4 seropositive NMOSD and MS
}

\author{
Yunyun Duan, ${ }^{1,2}$ Zhizheng Zhuo, ${ }^{1,2}$ Haiqing Li, ${ }^{3,4}$ De-Cai Tian (D) , 5,6,7 Yuxin Li, ${ }^{3,4}$ \\ Liqin Yang (D) , ${ }^{3,4}$ Chenyang Gao, ${ }^{5}$ Tian Zhang, ${ }^{8}$ Xinghu Zhang, ${ }^{5}$ Fu-Dong Shi (D) , 5,6,9 \\ Frederik Barkhof, ${ }^{10,11}$ Yaou Liu (1) ${ }^{1,2}$
}

- Additional material is published online only. To view, please visit the journal online (http://dx.doi.org/10.1136/ jnnp-2020-324826)

For numbered affiliations see end of article.

\section{Correspondence to} Professor Yaou Liu, Department of Radiology, Beijing Tiantan Hospital, Beijing 100070, China yaouliu80@163.com and Dr Haiqing Li, Department of Radiology, Huashan Hospital, FuDan University, Shanghai, 200040, China; haiqing226@ 163.com

YD and ZZ contributed equally.

$\mathrm{HL}$ and $\mathrm{YL}$ are joint senior authors.

Received 5 August 2020 Revised 7 December 2020 Accepted 1 February 2021 Published Online First 9 March 2021

\begin{abstract}
Background Brain structural alterations and their clinical significance of myelin oligodendrocyte glycoprotein antibody disease (MOGAD) have not been determined.

Methods We recruited 35 MOGAD, 38 aquaporin 4 antibody positive neuromyelitis optica spectrum diseases (AQP4+ NMOSD), 37 multiple sclerosis (MS) and 60 healthy controls $(\mathrm{HC})$ who underwent multimodal brain MRI from two centres. Brain lesions, volumes of the whole brain parenchyma, cortical and subcortical grey matter (GM), brainstem, cerebellum and cerebral white matter (WM) and diffusion measures (fractional anisotropy, FA and mean diffusivity, MD) were compared among the groups. Associations between the MRI measurements and the clinical variables were assessed by partial correlations. Logistic regression was performed to differentiate MOGAD from AQP4+ NMOSD and MS.

Results In MOGAD, 19 (54\%) patients had lesions on MRI, with cortical/juxtacortical (68\%) as the most common location. MOGAD and MS showed lower cortical and subcortical GM volumes than $\mathrm{HC}$, while AQP4+ NMOSD only demonstrated a decreased cortical GM volume. MS demonstrated a lower cerebellar volume, a lower FA and an increased MD than MOGAD and HC. The subcortical GM volume was negatively correlated with Expanded Disability Status Scale in MOGAD $(R=-0.51 ; p=0.004)$. A combination of MRI and clinical measures could achieve an accuracy of $85 \%$ and $93 \%$ for the classification of MOGAD versus AQP4+ NMOSD and MOGAD versus MS, respectively.

Conclusion MOGAD demonstrated cortical and subcortical atrophy without severe WM rarefaction. The subcortical GM volume correlated with clinical disability and a combination of MRI and clinical measures could separate MOGAD from AQP4+ NMOSD and MS.
\end{abstract}

\section{INTRODUCTION}

Myelin oligodendrocyte glycoprotein (MOG) antibody-associated disease (MOGAD) is a clinical syndrome characterised pathologically by oligodendrocyte damage and primary demyelination. Clinical manifestations include acute disseminated encephalomyelitis (ADEM), mostly in young children, and an opticospinal presentation in adults. Compared with other common demyelinating diseases including aquaporin 4 (AQP4) antibody seropositive neuromyelitis optica spectrum disorders (AQP4+ NMOSD) and multiple sclerosis (MS), MOGAD has distinct demographics, clinical features, prognosis and treatment options. Early accurate diagnosis of MOGAD is important to choose the optimal therapy and improve the prognosis. ${ }^{12}$

Characteristics of the lesion location (eg, brainstem) and morphology (ADEM-like or fluffy lesions) in MOGAD have been identified ${ }^{3-5}$ and proposed as imaging markers for differentiating MOGAD from AQP4+ NMOSD and MS, although the studies have not been concordant. Focal lesions are probably only the tip of the iceberg in terms of the pathological brain alterations; therefore, they are only weakly correlated with clinical disability. Grey matter (GM) atrophy reflecting neurodegeneration and white matter (WM) fibre integrity disruption reflecting demyelination are observed in AQP4+ NMOSD and MS with differential patterns, ${ }^{67}$ but the extent to which these compartments are also affected in MOGAD is unknown. Identifying the pattern of brain alterations in MOGAD can help in better understanding this unique disease and facilitate the development of objective imaging markers for monitoring disease progression and differentiating MOGAD from its mimics (AQP4+ NMOSD and MS).

We hypothesised that MOGAD might present with a distinct pattern of brain structural alterations compared with those of AQP4+ NMOSD and MS. Therefore, we conducted a prospective study aiming to evaluate brain lesions, regional volumetrics (cortical, subcortical, brainstem, cerebellum, WM) and WM diffusion measures in MOGAD using multimodal MRI, compared with AQP4+ NMOSD and MS, to establish the clinical significance of their differences.

\section{METHODS}

Standard protocol approvals, registrations and patient consent

The institutional review boards of Centre 1: Beijing Tiantan Hospital, Capital Medical University, Beijing, China (No. KY-2019-050-02) and Centre 2: Huashan Hospital, Fudan University, Shanghai, China (No. 2020-940) approved the study and written informed consent was obtained from each participant prior to participation. 


\section{Subjects}

The inclusion criteria for MOGAD were as follows: (1) at least one acute clinical demyelinating episode of the central nervous system (myelitis, optic neuritis or encephalopathy) persisting for a minimum of 24 hours; (2) MOG antibody seropositive by a cell-based assay (CBA) method; (3) AQP4-antibody negative; (4) an MRI scan at least 4 weeks after the attack; (5) age from 16 to 65 years to exclude the potential confounding factors of brain development under the age of 16 years and brain ageing above the age of 65 years. The exclusion criteria were (1) incomplete clinical assessment; (2) contradictions to MRI or a poor image quality or (3) a history of other neurological or neuropsychological diseases (eg, stroke or dementia). We recruited patients with AQP4 + NMOSD who were AQP4 antibody positive and patients with relapsing-remitting MS as disease controls and normal volunteers as healthy controls (HC). The NMOSD diagnosis was based on the 2015 International Panel on NMOSD Diagnosis, ${ }^{8}$ and all patients had antibodies against AQP4 using a CBA method. The MS diagnosis was determined according to the 2017 McDonald criteria ${ }^{9}$ with both MOG and AQP4 antibody seronegativity. Clinical variables including gender, age, disease duration, Expanded Disability Status Scale (EDSS) scores and the number of relapses were recorded.

\section{MRI images acquisition}

MRI images including fluid-attenuated inversion recovery imaging (FLAIR), 3D T1 and diffusion tensor imaging (DTI) were acquired based on 3.0 Tesla MR scanners (Philips Ingenia CX in centre 1 and GE Discovery MR750 in centre 2). Sagittal 3D FLAIR was performed using inversion recovering fast spin echo (IR-FSE) on Philips Ingenia CX (time of repetition (TR)/ time of echo $(\mathrm{TE})=4800 \mathrm{~ms} / 228 \mathrm{~ms}$, inversion time $(\mathrm{TI})=1650$ $\mathrm{ms}$, flip angle $(\mathrm{FA})=90^{\circ}$, image resolution $=1 \mathrm{~mm} \times 1 \mathrm{~mm} \times 1$ $\mathrm{mm}$ ), and axial 2D FLAIR were conducted using IR-FSE on a GE Discovery MR750 (TR/TE $=8800 \mathrm{~ms} / 150 \mathrm{~ms}, \mathrm{TI}=2100$ $\mathrm{ms}, \mathrm{FA}=111^{\circ}$, in-plane image resolution $=0.5 \mathrm{~mm} \times 0.5 \mathrm{~mm}$, slice thickness $=8 \mathrm{~mm}$,). Sagittal 3D T1 images were acquired using magnetisation-prepared rapid gradient echo on both a Philips Ingenia $C X\left(\mathrm{TR} / \mathrm{TE}=6.6 \mathrm{~ms} / 3 \mathrm{~ms}, \mathrm{TI}=880 \mathrm{~ms}, \mathrm{FA}=8^{\circ}\right.$, image resolution $=1 \mathrm{~mm} \times 1 \mathrm{~mm} \times 1 \mathrm{~mm})$ and GE Discovery MR750 (TR/TE $=8.2 \mathrm{~ms} / 3.2 \mathrm{~ms}, \mathrm{TI}=450 \mathrm{~ms}, \mathrm{FA}=12^{\circ}$, image resolution $=1 \mathrm{~mm} \times 1 \mathrm{~mm} \times 1 \mathrm{~mm})$. Axial $2 \mathrm{D}$ DTI images were obtained using spin echo-echo planar imaging on both a Philips Ingenia CX (TR/TE $=4000 \mathrm{~ms} / 88 \mathrm{~ms}, \mathrm{FA}=90^{\circ}$, in-plane image resolution $=2.5 \mathrm{~mm} \times 2.5 \mathrm{~mm}$, slice thickness $=2.5 \mathrm{~mm}$, slice gap $=0.25 \mathrm{~mm}, \mathrm{~b}$ values $=0$ and $1000 \mathrm{~s} / \mathrm{mm}^{2}$, number of the diffusion sensitive gradient direction $=48$ ) and a GE Discovery MR750 (TR/TE $=4700 \mathrm{~ms} / 98 \mathrm{~ms}, \mathrm{FA}=90^{\circ}$, in-plane image resolution $=2 \mathrm{~mm} \times 2 \mathrm{~mm}$, slice thickness $=4 \mathrm{~mm}$, slice gap $=0.4 \mathrm{~mm}, \mathrm{~b}$ values $=0$ and $1000 \mathrm{~s} / \mathrm{mm}^{2}$, number of the diffusion sensitive gradient direction $=50$ ).

\section{Image analysis}

All of the MRI scans were reviewed, and the lesions were outlined by an experienced neuroradiologist (YD, with 12 years' experience in neuroradiology) using 3D Slicer software (https://www.slicer.org/) on the FLAIR images. Prior to the segmentations, the 3D T1 images were lesion-filled using the Lesion Segmentation Tool (V.3.0.0, https://www.applied-statistics.de/lst.html). Segmentation was performed by Computational Anatomy Toolbox (Cat) in Statistical Parametric Mapping (SPM12, https:/www.fil.ion.ucl.ac.uk/spm/). Brain parenchyma volume, cortical and subcortical GM (including the bilateral thalamus, caudate, putamen, pallidum, hippocampus, amygdala, accumbens area and ventral diencephalon), brainstem and cerebellar volumes and the cerebral WM volume were obtained based on the neuromorphometrics atlas (http:// www.neuromorphometrics.com/). DTI was processed using the FMRIB Software Library (FSL V.6.0, https://fsl.fmrib.ox. ac.uk/fsl/fslwiki/FSL), including eddy-current and motion artefact correction, skull removal and DTI tensor fitting. Average fractional anisotropy (FA) and mean diffusivity (MD) within the fibre-tracts were extracted based on the JHU fibre-tract atlas. $^{10}$

\section{Statistical analyses}

Statistical analyses were performed by using SPSS software (V.22; SPSS, Chicago, Illinois, USA), MATLAB Statistics and the Machine Learning Toolbox (MATLAB 2019). Categorical data are presented in percentages. Continuous data are presented by the mean and SD, and ranked data by the median and IQR. Categorical data between groups were analysed by the $\chi^{2}$ test. Continuous and ranked data were analysed by oneway analysis of variance or Kruskal-Wallis tests followed by posthoc comparisons with Bonferroni correction. For MRI quantitative measures (except for the lesion volumes), total intracranial volume (TIV, only used for the volume measures), gender, age and scanner type were included as covariates. Partial correlation was performed to investigate the relationship between the MRI measures and the clinical variables, including the disease duration, EDSS and number of relapses with covariate adjustment including TIV (only used for volume measures), gender, age and scanner type. Logistic regression was conducted to identify factors distinguishing MOGAD from AQP4 + NMOSD and MS by using MRI measures and clinical variables (disease duration, EDSS and the number of relapses) with TIV, gender, age and scanner type as confounding variables. The classification accuracy, sensitivity, specificity and area under the curve (AUC) were calculated.

\section{Sensitivity analysis}

Sensitivity analyses were carried out in (1) subsamples of female subjects to exclude any gender effect, (2) subsamples with a disease duration of less than 3 years to exclude any effects of disease duration and to investigate the early changes, (3) subsamples with normal brain MRI to exclude the lesion effect on the GM/WM measures and (4) subsamples in a single centre (centre 1) to exclude potential recruitment bias.

\section{RESULTS}

\section{Demographics and clinical characteristics}

Thirty-seven patients with MOGAD (17 from centre 1 and 20 from centre 2), 39 patients with AQP4 + NMOSD (21 from centre 1 and 18 from centre 2) who were AQP4 antibody positive and 39 patients with relapsing-remitting MS (15 from centre 1 and 24 from centre 2 ) and $63 \mathrm{HC}$ (30 from centre 1 and 33 from centre 2) were recruited for this study. None of the $\mathrm{HC}$ had a current or previous history of neurological dysfunction and none had MRI visible abnormalities. Three patients (one MOGAD and two MS) and three HC were excluded due to poor image quality, and one patient with MOGAD and one patient with AQP4 + NMOSD were excluded due to a history of other neurological diseases (cerebral trauma and incidental meningioma, respectively). The final sample consisted of 170 subjects including 35 MOGAD (21 women; mean age (SD) 
36.4 (13.6) years), 38 AQP4 + NMOSD (32 women; 37.7 (11.9) years), 37 MS (23 women; 33.8 (11.2) years) and 60 HC (31 women; 36.9 (12.7) years).

No significant differences were observed in age among the MOGAD, AQP4+ NMOSD, MS and HC. A higher female/ male ratio was observed in AQP4 + NMOSD (84\%) compared with MOGAD (60\%; $\mathrm{p}=0.02)$, MS (62\%; $\mathrm{p}=0.02)$ and HC $(52 \% ; p=0.001)$, while the other groups were similar. The most common symptom in MOGAD was optic neuritis (94\%), similar to AQP4 + NMOSD (82\%), but higher than in MS (32\%). No differences were identified in disease duration among MOGAD (median, 0.8 years), AQP4+ NMOSD (1.4 years) and MS (3 years). AQP4 + NMOSD had a higher median EDSS than MS (3.5 vs 2.5; p=0.003). No significant differences in EDSS were observed between MOGAD (median, 3) and AQP4+ NMOSD or between MOGAD and MS (table 1). Additionally, 29\% MOGAD $(\mathrm{n}=10)$ and $16 \%$ AQP4+ NMOSD $(n=6)$ were monophasic. No significant differences in the number of relapses among the groups were observed.

\begin{tabular}{|c|c|c|c|c|}
\hline & $\mathrm{HC}(\mathrm{n}=60)$ & MOGAD $(n=35)$ & AQP4+ NMOSD $(n=38)$ & MS $(n=37)$ \\
\hline \multicolumn{5}{|l|}{ Clinical characteristic } \\
\hline Gender, female ratio (\%) & $31 / 60(52 \%)$ & $21 / 35(60 \%)^{*}$ & $32 / 38(84 \%)+\ddagger$ & $23 / 37(62 \%)$ \\
\hline Age (mean (SD), year) & $36.9(12.7)$ & $36.4(13.6)$ & $37.7(11.9)$ & $33.8(11.2)$ \\
\hline Disease duration (median (IQR), year) & & $0.8(0.3-4.3)$ & $1.4(0.8-3.2)$ & $3(1-5.3)$ \\
\hline EDSS (median (IQR)) & & $3(2-4)$ & $3.5(2-4.5) \dagger$ & $2.5(1-3)$ \\
\hline Monophasic, n (\%) & & $10(29 \%) \dagger$ & $6(16 \%)+$ & $0(0 \%)$ \\
\hline Number of relapses (median (IQR)) & & $2(1-4)$ & $2(2-3)$ & $2(2-4)$ \\
\hline \multicolumn{5}{|l|}{ Clinical symptom } \\
\hline Optic neuritis, n (\%) & & $33(94 \%) \dagger$ & $31(82 \%) \dagger$ & $12(32 \%)$ \\
\hline Myelitis, n (\%) & & $10(29 \%)^{*}$ & $28(74 \%) \dagger$ & $12(32 \%)$ \\
\hline Encephalopathy, n (\%) & & $3(9 \%)$ & $2(7 \%)$ & $2(5 \%)$ \\
\hline \multicolumn{5}{|l|}{ Brain lesions } \\
\hline Presence of brain lesions, $\mathrm{n}(\%)$ & & $19(54 \%)+$ & $23(61 \%) \dagger$ & $36(97 \%)$ \\
\hline Cortical/juxtacortical, n (\%) & & $13(68 \%)^{*}$ & $6(26 \%) \dagger$ & $29(81 \%)$ \\
\hline Deep WM, n (\%) & & $9(47 \%)+$ & $10(43 \%)+$ & $35(96 \%)$ \\
\hline $\begin{array}{l}\text { Periventricular WM, } \\
\mathrm{n}(\%)\end{array}$ & & $11(58 \%) \dagger$ & $12(52 \%)+$ & $35(96 \%)$ \\
\hline Corpus callosum, $\mathrm{n}(\%)$ & & $3(16 \%)$ & $4(17 \%)$ & $19(53 \%)$ \\
\hline Basal ganglia, $n(\%)$ & & $4(21 \%)$ & $5(22 \%)$ & $12(33 \%)$ \\
\hline Thalamus, $n(\%)$ & & $1(5 \%)$ & $4(17 \%)$ & $7(19 \%)$ \\
\hline Internal capsule, $\mathrm{n}(\%)$ & & $4(21 \%)$ & $4(17 \%)$ & $8(22 \%)$ \\
\hline Brain stem, $\mathrm{n}(\%)$ & & $6(32 \%)$ & $7(30 \%)$ & $15(42 \%)$ \\
\hline Midbrain, $n(\%)$ & & $5(26 \%)$ & $5(22 \%)$ & $3(8 \%)$ \\
\hline Pontine, n (\%) & & $2(11 \%)$ & $2(9 \%) \dagger$ & $11(31 \%)$ \\
\hline Medulla oblongata, n (\%) & & $3(16 \%)$ & $4(17 \%)$ & $4(11 \%)$ \\
\hline Cerebellar peduncles, $n(\%)$ & & $1(5 \%)$ & $1(4 \%)$ & $6(17 \%)$ \\
\hline Cerebellum, n (\%) & & $0 \dagger$ & $0+$ & $6(17 \%)$ \\
\hline NMOSD-specific lesions, $\mathrm{n}(\%)$ & & $2(11 \%)$ & $4(17 \%)$ & $1(3 \%)$ \\
\hline Periependymal surfaces of third ventricle, $n(\%)$ & & 0 & $1(4 \%)$ & 0 \\
\hline Periependymal surfaces of fourth ventricle, $\mathrm{n}(\%)$ & & $2(11 \%)$ & $3(13 \%)$ & $1(3 \%)$ \\
\hline Area postrema, $\mathrm{n}(\%)$ & & $1(5 \%)$ & $1(4 \%)$ & 0 \\
\hline \multicolumn{5}{|l|}{ MS-specific lesions, $n(\%)$} \\
\hline $\begin{array}{l}\text { Lesions adjacent to the body of the lateral ventricle and in the inferior temporal } \\
\text { lobe, } n(\%)\end{array}$ & & $0 \dagger$ & $0+$ & $14(39 \%)$ \\
\hline Dawson's finger type lesions, $\mathrm{n}(\%)$ & & $0+$ & $1(4 \%) \dagger$ & $13(36 \%)$ \\
\hline $\mathrm{S}$ or U shape lesions, $\mathrm{n}(\%)$ & & $2(11 \%)$ & $3(13 \%)$ & $11(31 \%)$ \\
\hline \multicolumn{5}{|l|}{ Quantitative MRI measures } \\
\hline Lesion volume (mean (SD), mL) & & $9.1(18.2)$ & $2.5(3.1)$ & $16.5(15.7)$ \\
\hline Brain parenchyma volume (mean (SD), mL) & $1128.2(31.4)$ & $1095.2(66.2) \dagger \ddagger$ & $1100.8(53.6) \dagger$ & $1051.6(70.5) \ddagger$ \\
\hline Cortical GM volume (mean (SD), mL) & $509.8(20.0)$ & $493.5(30.0) \ddagger$ & $493.7(28.1) \ddagger$ & $483.5(24.8) \ddagger$ \\
\hline Subcortical GM volume (mean (SD), mL) & $40.9(1.9)$ & $39.0(3.3) \dagger \ddagger$ & $39.4(2.7) \dagger$ & $34.6(5.6) \ddagger$ \\
\hline Brainstem volume (mean (SD), $\mathrm{mL}$ ) & $16.7(1.1)$ & $16.2(1.2)$ & $16.5(1.2)$ & $15.7(1.7) \ddagger$ \\
\hline Cerebellum volume (mean (SD), $\mathrm{mL}$ ) & $125.7(8.2)$ & $121.8(7.3) \dagger$ & $120.3(8.1) \ddagger$ & $116.4(9.7) \ddagger$ \\
\hline Cerebral WM volume (mean (SD), mL) & $419.6(17.9)$ & $408.2(33.9) \dagger$ & $413.3(25.7) \dagger$ & $385.0(42.4) \ddagger$ \\
\hline FA (mean (SD)) & $0.47(0.02)$ & $0.46(0.02) \dagger$ & $0.46(0.02) \dagger$ & $0.43(0.02) \ddagger$ \\
\hline $\mathrm{MD}\left(\right.$ mean $\left.(\mathrm{SD}), 10^{-3} \mathrm{~mm}^{2} / \mathrm{s}\right)$ & $0.92(0.03)$ & $0.94(0.04) \dagger$ & $0.93(0.03) t$ & $0.99(0.05) \ddagger$ \\
\hline \multicolumn{5}{|c|}{$\begin{array}{l}\text { MRI measurements (except for lesion volume) were adjusted for total intracranial volume (only for volume measurements), gender, age and scanner type. } \\
\text { Statistical significance with two-sided p<0.05. } \\
\text { *Indicated a statistically significant difference compared with AQP4+ NMOSD. } \\
\text { tIndicated a statistically significant difference compared with MS. } \\
\text { fIndicated a statistically significant difference compared with HC. } \\
\text { AQP4+ NMOSD, aquaporin } 4 \text { seropositive neuromyelitis optica spectrum disorders; EDSS, expanded disability status scale; FA, fractional anisotropy; GM, grey matter; HC, healthy controls; MD, mean diffusivity; MOGAD, } \\
\text { myelin oligodendrocyte glycoprotein antibody associated disease; MS, multiple sclerosis; WM, white matter. }\end{array}$} \\
\hline
\end{tabular}




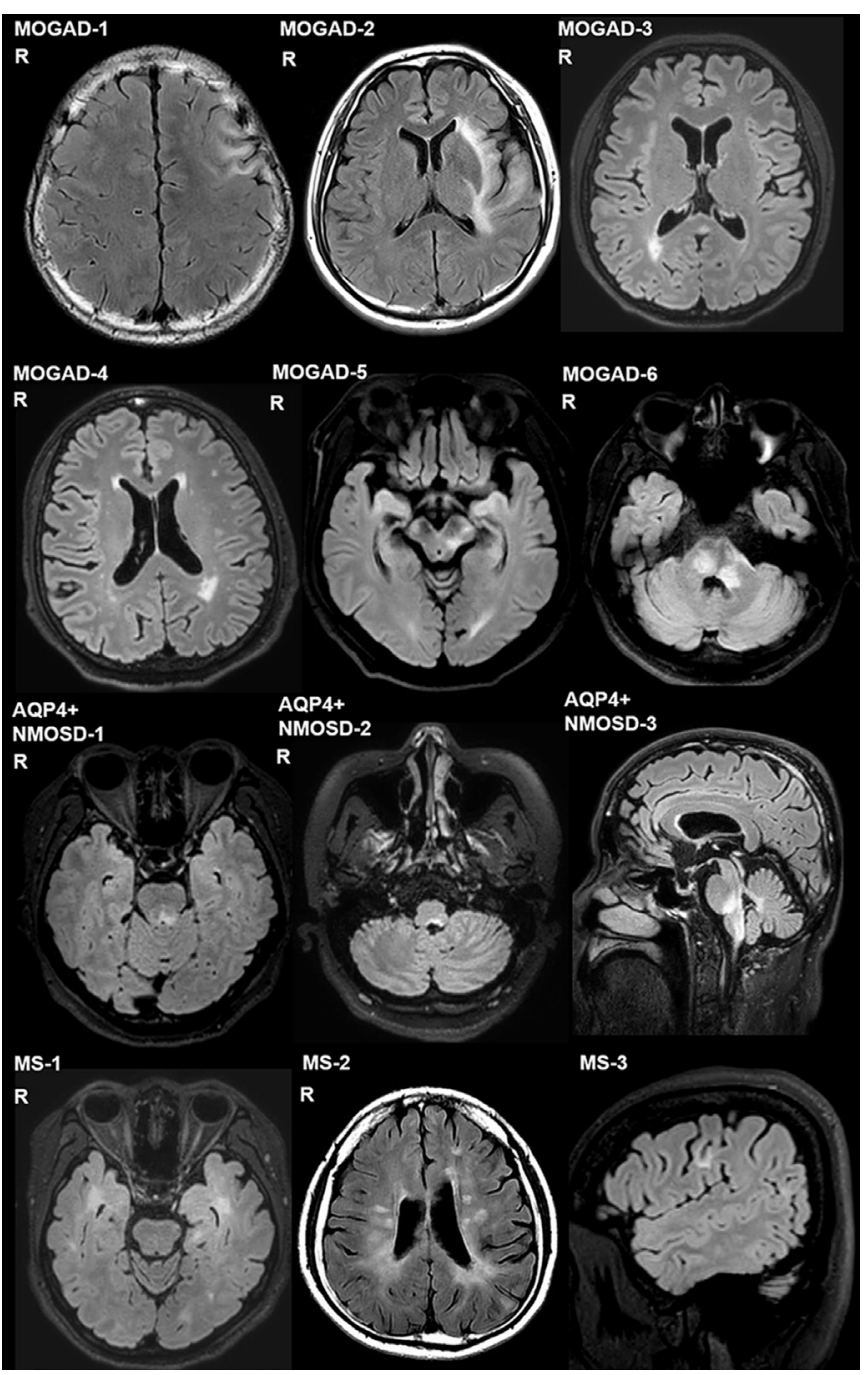

Figure 1 FLAIR images of representative cases of MOGAD, AQP4+ NMOSD and MS. MOGAD brain lesions: cortical grey matter/juxtacortical white matter (MOGAD-1, 2), periventricular white matter (MOGAD-3, 4), deep white matter (MOGAD-4), midbrain (MOGAD-5) and pontine and periependymal surfaces of the fourth ventricle (MOGAD-6). AQP4+ NMOSD brain lesions: midbrain periaqueductal area (AQP4+ NMOSD-1), periependymal surfaces of the fourth ventricle and the area postrema (AQP4+ NMOSD-2, 3). MS brain lesions: inferior temporal lobe (MS-1), Dawson's finger-type lesions (MS-2) and S or U shape lesions (MS-3). AQP4+ NMOSD, aquaporin 4 antibody positive neuromyelitis optica spectrum diseases; FLAIR, fluid-attenuated inversion recovery imaging; MOGAD, myelin oligodendrocyte glycoprotein antibody disease; MS, multiple sclerosis; $\mathrm{R}$, right.

\section{Lesion characteristics}

As shown in table 1, 19 (54\%) patients with MOGAD, 23 (61\%) patients with AQP4+ NMOSD and 36 (97\%) patients with MS presented with brain MRI lesions. In MOGAD, the most frequent lesion location was cortical/juxtacortical (68\%), similar to MS $(82 \%)$, but significantly more frequent than in AQP4+ NMOSD $(26 \% ; \mathrm{p}<0.001)$ (table 1). The other common lesion locations in MOGAD (figure 1) were periventricular WM (58\%), supratentorial deep WM (47\%), brainstem (32\%), basal ganglia (21\%) and corpus callosum (16\%).

There was no significant difference in the presence of NMOSDspecific periependymal or area postrema lesions between MOGAD $(11 \%)$ and AQP4+ NMOSD $(17 \%)(p=0.29)$. For MS specific lesions, lesions adjacent to the body of the lateral ventricle and in the inferior temporal lobe, and Dawson finger lesions, ${ }^{11}$ were not observed in MOGAD and only appeared in one patient with AQP4+ NMOSD, but presented in 14 (39\%) and 13 (36\%) patients with MS, respectively. S or U shape lesions were observed in $2(11 \%)$ MOGAD, showing no significant difference with AQP4+ NMOSD (13\%) and MS (31\%). For lesion volume measures, MOGAD (mean (SD), 9.1 (18.2) mL; p=0.008) and AQP4+ NMOSD (2.5 (3.1) $\mathrm{mL} ; \mathrm{p}<0.001)$ presented with smaller lesion volumes than MS $(16.5$ (15.7) $\mathrm{mL}$ ).

\section{Structural MRI measures}

All brain volume and diffusion measures of MOGAD, AQP4+ NMOSD, MS and HC are shown in table 1 and figure 2. For brain volume measurements, MOGAD (1095.2 (66.2) mL; p=0.03) and MS (1051. $6(70.5) \mathrm{mL} ; \mathrm{p}<0.001)$ but not AQP4+ NMOSD (1100.8 (53.6) $\mathrm{mL} ; \mathrm{p}=0.09)$ showed a decreased brain parenchyma volume compared with HC (1128.2 (31.4) mL), while MS showed a lower brain parenchyma volume than MOGAD $(\mathrm{p}=0.005)$. A decreased cortical GM volume was found in MOGAD (493.5 (30.0) $\mathrm{mL}$; $\mathrm{p}=0.02)$, AQP4 + NMOSD (493.7 (28.1) $\mathrm{mL} ; \mathrm{p}=0.02)$ and MS (483.5 (24.8) $\mathrm{mL} ; \mathrm{p}<0.001)$ compared with HC $(509.8$ (20.0) $\mathrm{mL}$ ), while MOGAD showed no significant differences from AQP4+ NMOSD $(\mathrm{p}=0.99)$ and MS $(\mathrm{p}=0.56)$. MOGAD (39.0 (3.3) $\mathrm{mL} ; \mathrm{p}=0.005)$ and MS (34.6 (5.6) mL; $\mathrm{p}<0.001)$ but not AQP4+ NMOSD (39.4 (2.7) mL; p=0.26) showed a decreased subcortical GM volume compared with HC (40.9 (1.9) mL), while MS demonstrated a lower subcortical GM volume than MOGAD ( $\mathrm{p}<0.001)$. For brainstem and cerebellar volumes, there were no significant differences between MOGAD (brainstem: 16.2 (1.2) $\mathrm{mL} ; \mathrm{p}=0.45$, cerebellum: 121.8 (7.3) $\mathrm{mL} ; \mathrm{p}=0.16$ ) and $\mathrm{HC}$ (brainstem:16.7 (1.1) mL, cerebellum: 125.7 (8.2) mL), while MS (116.4 (9.7) mL; $\mathrm{p}=0.04$ ) had a lower cerebellar volume compared with MOGAD. Concerning cerebral WM volume, there were no significant differences among MOGAD (408.2 (33.9) mL), AQP4+ NMOSD (413.3 (25.7) $\mathrm{mL}$ ) and HC (419.6 (17.9) mL), while MS (385.0 (42.4) mL) showed a decreased volume compared with MOGAD $(p=0.007)$.

\section{Diffusion MRI measures}

For the diffusion measurements, MOGAD (FA: 0.46 (0.02), MD: $\left.0.94(0.03) \times 10^{-3} \mathrm{~mm}^{2} / \mathrm{s}\right)$ showed no significant difference in FA or MD compared with HC (FA: $0.47(0.02) ; \mathrm{p}=0.79$, MD: 0.92 $\left.(0.03) \times 10^{-3} \mathrm{~mm}^{2} / \mathrm{s} ; \mathrm{p}=0.05\right)$ or $\mathrm{AQP} 4+\mathrm{NMOSD}$ (FA: 0.46 (0.02); $\left.\mathrm{p}=1, \mathrm{MD}: 0.93(0.03) \times 10^{-3} \mathrm{~mm}^{2} / \mathrm{s} ; \mathrm{p}=0.43\right)$, while MS demonstrated a lower FA $(0.43(0.02) ; \mathrm{p}<0.001)$ and a higher MD $\left(0.99(0.05) \times 10^{-3} \mathrm{~mm}^{2} / \mathrm{s} ; \mathrm{p}=0.004\right)$ than MOGAD and HC.

Correlation of MRI characteristics with clinical variables

Differential patterns of MRI-clinical correlations were observed in MOGAD, AQP4+ NMOSD and MS (figure 3). For MOGAD, the subcortical GM volume was negatively correlated with EDSS $(\mathrm{R}=-0.51,95 \% \mathrm{CI},-0.63$ to -0.45$) \mathrm{p}=0.004)$ and the number of relapses ( $\mathrm{R}=-0.47,95 \% \mathrm{CI},-0.51$ to $-0.24 ; \mathrm{p}=0.008)$, and FA was negatively correlated with disease duration $(\mathrm{R}=-0.36$, $95 \% \mathrm{CI},-0.46$ to -0.29$) ; \mathrm{p}=0.05)$ and the number of relapses $(\mathrm{R}=-0.38,95 \% \mathrm{CI},-0.53$ to $-0.27 ; \mathrm{p}=0.03)$. For $\mathrm{AQP} 4+$ NMOSD, FA ( $\mathrm{R}=-0.44,95 \% \mathrm{CI},-0.62$ to -0.44$) ; \mathrm{p}=0.01)$ was negatively correlated with EDSS, and no MRI measures correlated with disease duration. For MS, subcortical GM $(\mathrm{R}=-0.42,95 \% \mathrm{CI}$, -0.41 to -0.14$) ; \mathrm{p}=0.02)$, brain stem $(-0.45,95 \% \mathrm{CI},-0.48$ to $-0.07 ; \mathrm{p}=0.01)$, cerebral WM volume $(\mathrm{R}=-0.39,95 \% \mathrm{CI},-0.31$ to -0.02$) ; \mathrm{p}=0.03)$ and $\mathrm{MD}(\mathrm{R}=0.35,95 \% \mathrm{CI}, 0.17$ to 0.52$)$; $\mathrm{p}=0.05)$ were correlated with EDSS. Lesion volume $(\mathrm{R}=0.37,95 \%$ 

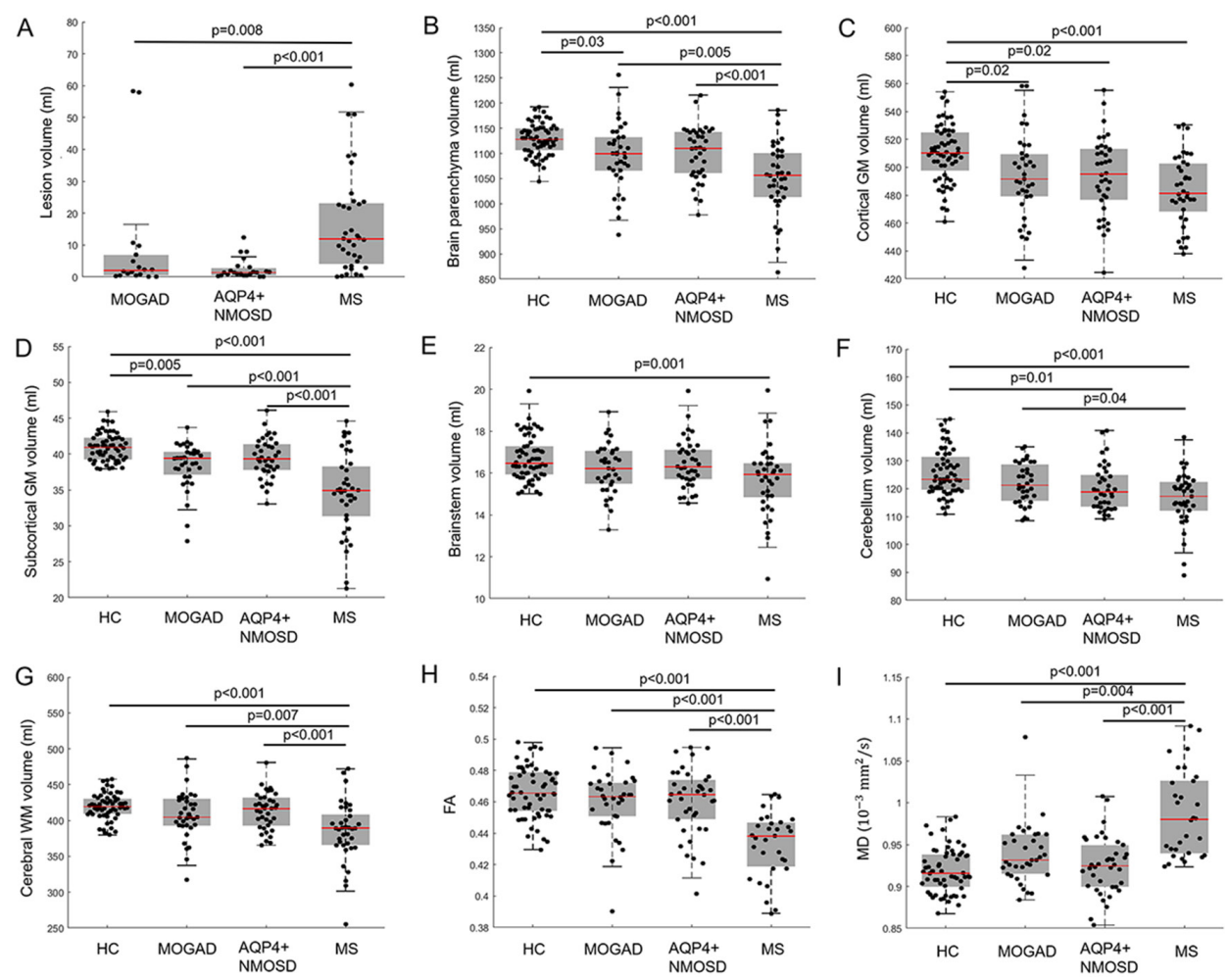

Figure 2 Structural MRI measures between MOGAD, AQP4+ NMOSD, MS and HC. Structural MRI measures including (A) lesion volume, (B) brain parenchyma volume, (C) cortical GM volume, (D) subcortical GM volume, (E) brainstem volume, (F) cerebellar volume, (G) cerebral WM volume, (H) FA and (I) MD among HC, MOGAD, AQP4+ NMOSD and MS. Statistical analyses were performed using one-way ANOVA or Kruskal-Wallis tests followed by posthoc comparisons with Bonferroni correction. MRI measurements (except for lesion volume) were adjusted for total intracranial volume (only for the volume measurements), gender, age and scanner type. Significance with two-sided $p<0.05$ were considered. ANOVA, analysis of variance; AQP4+ NMOSD, aquaporin four antibody positive neuromyelitis optica spectrum diseases; FA, fractional anisotropy; GM, grey matter; HC, healthy controls; MD, mean diffusivity; MOGAD, myelin oligodendrocyte glycoprotein antibody disease; MS, multiple sclerosis; WM, white matter.

$\mathrm{CI}, 0.25$ to 0.46$) ; \mathrm{p}=0.03)$ and brain stem volume $(\mathrm{R}=-0.36,95 \%$ $\mathrm{CI},-0.42$ to $-0.27 ; \mathrm{p}=0.05$ ) were correlated with disease duration.

\section{Classification using MRI measures}

Univariate logistic regression demonstrated that a few MRI and clinical measures were able to differentiate MOGAD, AQP4+ NMOSD and MS (online supplemental eTable 1). Due to the collinearity of the MRI measures (online supplemental eFigures 5 and 6), a forward stepwise logistic regression was carried out for differentiating the diagnosis of MOGAD versus AQP4+ NMOSD, MOGAD vs AQP4+ NMOSD in subgroups without brain lesions, and MOGAD versus MS, respectively, with cofounding factors of TIV, gender, age and scanner type. For MOGAD versus AQP4+ NMOSD, the classification achieved an accuracy of $85 \%$, sensitivity of $80 \%$, specificity of $89 \%$ and AUC of 0.89 by using cerebellar volume. For the patients without brain lesions, the classification achieved an accuracy of $81 \%$, sensitivity of $76 \%$, specificity of $87 \%$ and AUC of 0.88 by using cerebellar volume. For MOGAD versus MS, the classification achieved an accuracy of 93\%, sensitivity of $89 \%$, specificity of $97 \%$ and AUC of 0.97 by FA (table 2 and figure 4).

\section{Sensitivity analysis results}

The sensitivity analysis results with (1) subsamples who were women (online supplemental eFigure 1), (2) subsamples in the early phases (online supplemental eFigure 2), (3) subsamples without brain lesions (online supplemental eFigure 3), and (4) subsamples from a single centre (centre 1) (online supplemental eFigure 4) showed that most findings of the whole group analysis were preserved in the subgroups, indicating the findings were not significantly altered when restricted to these subgroups.

\section{DISCUSSION}

In this study, we demonstrated that the lesion distribution and brain structural alteration patterns of early MOGAD (70\% patients with a disease duration less than 3 years) differed from AQP4+ NMOSD and MS. MOGAD lesions were predominantly located in the cortical/juxticortical area, and the patients presented with cortical and subcortical GM atrophy without severe WM atrophy or rarefaction. AQP4+ NMOSD showed less subcortical GM atrophy and MS showed more brain atrophy in both the GM and WM components along with severe WM rarefaction as compared with MOGAD. Subcortical GM volume correlated with clinical disability in MOGAD, which was different from AQP4+ NMOSD and MS. MRI measures could distinguish MOGAD, AQP4+ NMOSD and MS, even in patients with MOGAD and AQP4+ NMOSD without visible brain lesions.

Consistent with previous reports of predominantly AQP4+ NMOSD phenotypes, ${ }^{12-16}$ we confirmed a high predilection for optic nerve and spinal cord involvement in adult MOGAD. We found that $54 \%$ of patients with MOGAD presented abnormalities on brain MRI, which is consistent with previous studies showing the presence of brain lesions in MOGAD ranging from 24\% to 83\%.5 $121316-18$ The most common lesion location in MOGAD was cortical/juxtacortical (68\%), followed by periventricular (58\%), deep WM (47\%) and brainstem (particularly midbrain), comparable to previous findings, ${ }^{19-22}$ suggesting a possible relatively specific lesion pattern 
A

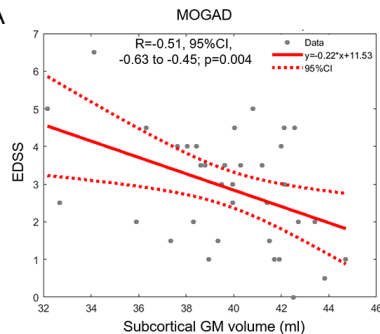

D

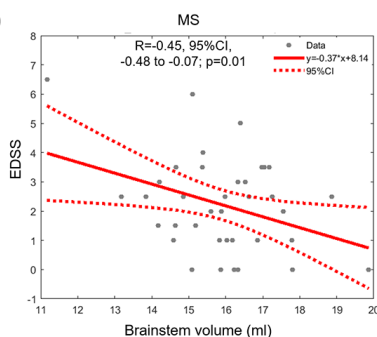

G
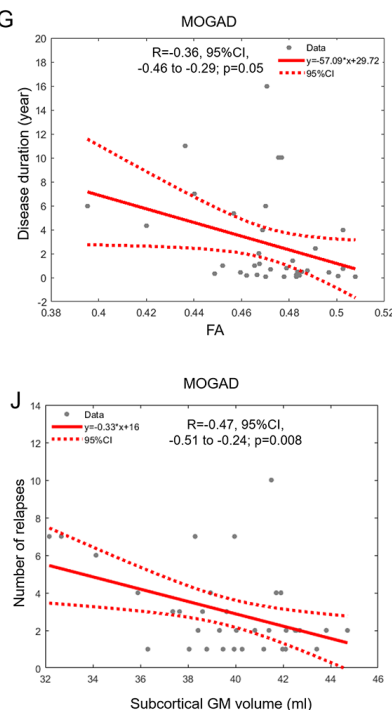

B

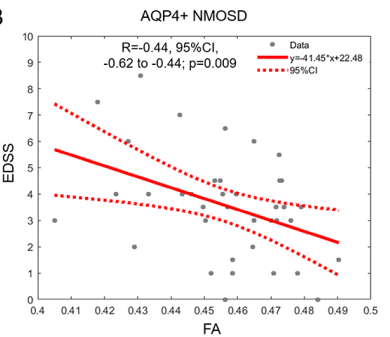

E

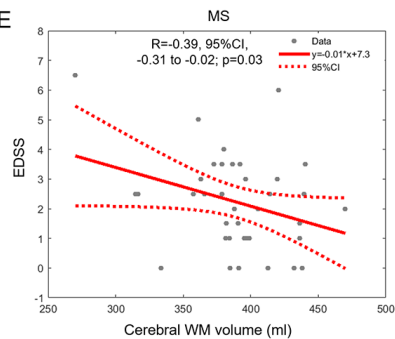

$\mathrm{H}$
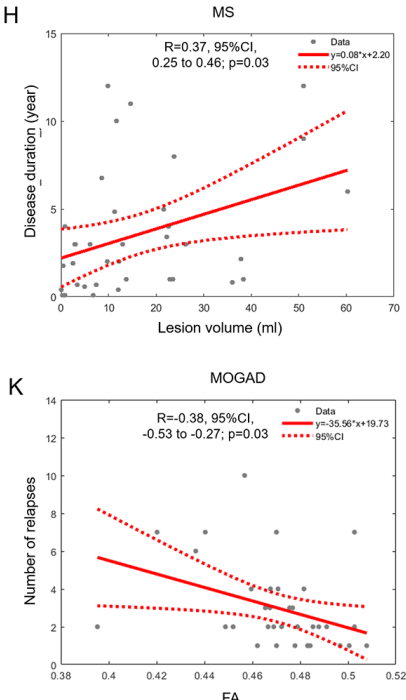
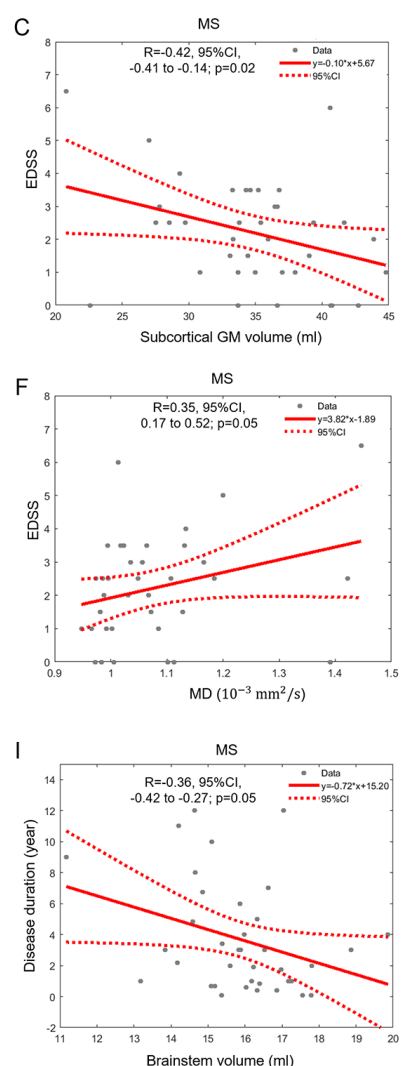

Brainstem volume (ml)

Figure 3 Differential correlation patterns with EDSS, disease duration and the number of relapses. (A-F) MRI measures correlating with EDSS in AQP4+ MOGAD (A) AQP4+ NMOSD (B) and MS (C-F); (G-I) MRI measures correlating with disease duration in MOGAD (G) and MS (H, I); (J-K) MRI measures correlating with the number of relapses in MOGAD. Partial correlation analyses were performed with significance (two-sided) at $\mathrm{p}<0.05$. AQP4+ NMOSD, aquaporin four antibody positive neuromyelitis optica spectrum diseases; EDSS, Expanded Disability Status Scale; FA, fractional anisotropy; GM, grey matter; MD, mean diffusivity; MOGAD, myelin oligodendrocyte glycoprotein antibody disease; MS, multiple sclerosis; WM, white matter.

in MOGAD, which should be further validated. Cortical/juxtacortical lesions but not AQP4+ NMOSD-specific lesions significantly differed between MOGAD and AQP4+ NMOSD, highlighting the role of cortical involvement in MOGAD in contrast to AQP4+ NMOSD, which is devoid of cortical lesions. ${ }^{5} 1222$ There was a consistent absence of MS specific lesions in the antibody-mediated diseases (MOGAD and AQP4+ NMOSD), consistent with previous studies, ${ }^{5} 23$ implying MS-specific lesions as a "red flag" MRI sign in MOGAD and AQP4+ NMOSD.

Most of the previous studies have been focusing on the lesion characteristics, ${ }^{3} 15$ and we innovatively identified brain atrophy and diffusion alterations in MOGAD. Cortical and subcortical GM atrophy was observed in patients with MOGAD, similar to a pattern seen in MS. We speculated that as in MS and NMOSD,

Table 2 Classification of MOGAD, AQP4+ NMOSD and MS by stepwise logistic regression

\begin{tabular}{|c|c|c|c|c|c|c|c|c|c|}
\hline \multirow{2}{*}{ Classification } & \multirow{2}{*}{ Feature } & \multirow[b]{2}{*}{ OR } & \multicolumn{2}{|c|}{$95 \% \mathrm{Cl}$} & \multirow[b]{2}{*}{$P$ value } & \multirow[b]{2}{*}{ Accuracy (\%) } & \multirow{2}{*}{$\begin{array}{l}\text { Sensitivity } \\
(\%)\end{array}$} & \multirow{2}{*}{$\begin{array}{l}\text { Specificity } \\
(\%)\end{array}$} & \multirow[b]{2}{*}{ AUC } \\
\hline & & & Lower bound & Upper bound & & & & & \\
\hline MOGAD vs AQP4+ NMOSD & Cerebellar volume & 1.14 & 1.11 & 1.19 & 0.009 & 85 & 80 & 89 & 0.89 \\
\hline $\begin{array}{l}\text { MOGAD vs AQP4+ NMOSD } \\
\text { (without brain lesions) }\end{array}$ & Cerebellar volume & 1.17 & 1.12 & 1.30 & 0.042 & 81 & 76 & 87 & 0.88 \\
\hline MOGAD vs MS & FA & $1.9 \times 10^{30}$ & $8.49 \times 10^{26}$ & $5.74 \times 10^{51}$ & 0.001 & 93 & 89 & 97 & 0.97 \\
\hline
\end{tabular}


A

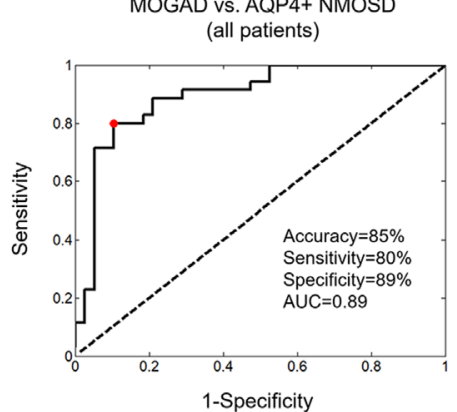

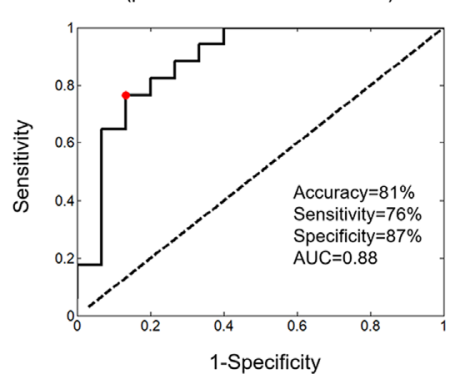

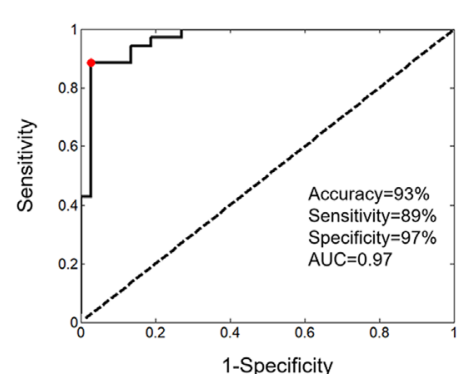

Figure 4 Classification performance by clinical and MRI measures using logistic regression. ROC of (A) MOGAD vs AQP4+ NMOSD (all patients), (B) MOGAD vs AQP4+ NMOSD (patients without brain lesions) and (C) AQP4+ MOGAD vs. MS are presented with classification accuracy, sensitivity, specificity and AUC. AQP4+ NMOSD, aquaporin 4 antibody positive neuromyelitis optica spectrum diseases; AUC, area under the curve; EDSS, Expanded Disability Status Scale; MOGAD, myelin oligodendrocyte glycoprotein antibody disease; MS, multiple sclerosis; ROC, receiver operating characteristic curve.

WM lesions might lead to neuron loss in the GM (eg, Wallerian degeneration) in MOGAD (online supplemental eFigures 7 and 8). ${ }^{24}$ Other mechanisms include reactive astrocytes and terminal complement activation. ${ }^{25}{ }^{26}$ Although GM atrophy was identified in all three inflammatory demyelinating diseases, the degree was different. MS showed more severe GM atrophy, particularly subcortical GM atrophy, than MOGAD and AQP4+ NMOSD, while AQP4+ NMOSD showed the mildest subcortical GM atrophy. Although brainstem lesions were present in MOGAD, brainstem and cerebellar volumes were not significantly reduced in MOGAD, suggesting infratentorial structures may be largely preserved in early MOGAD, which is different from previous findings in $\mathrm{NMOSD}^{27}$ and MS. ${ }^{28}$

WM atrophy and rarefaction (as shown by FA and MD) was not observed in MOGAD, similar to AQP4+ NMOSD, while MS showed severe WM atrophy and rarefaction. The differences between the WM and GM alterations in MOGAD and AQP4+ NMOSD suggest that there may be different injury mechanisms for these two brain compartments in these two antibody-mediated diseases, such as different expression patterns of the different isoforms of MOG or AQP4 or differential blood brain barrier permeability between GM and WM..$^{29-31}$ The WM alteration of MOGAD still needs to be further investigated by analysis at the regional level or tract level to identify the specific demyelinating fibres since demyelination is a pathological hallmark of MOGAD. ${ }^{32}$

Differential clinical-MRI correlations were observed between the three diseases. In MOGAD, clinical disability and the number of relapses were correlated with the subcortical GM volume, implying that this measure may serve as a potential biomarker for monitoring the disease progression and outcome in MOGAD clinical trials. In addition to GM atrophy, MS showed other MRI-clinical predictors, including WM volume, FA and $\mathrm{MD}$, while the only significant correlation with EDSS was with FA in AQP4+ NMOSD.

For discriminating MOGAD, AQP4+ NMOSD and MS, the lesion distribution can contribute to the differential diagnosis; ${ }^{523}$ however, approximately $50 \%$ of patients with early MOGAD and AQP4+ NMOSD do not present with focal brain lesions. Quantitative MRI measures (eg, cerebellar volume) can classify MOGAD versus AQP4+ NMOSD with good accuracy (85\%). Interestingly, in patients without brain lesions, the accuracy is maintained (81\%), implying the degrees of cerebellar volume as good indices for discriminating MOGAD from AQP4+ NMOSD. Using FA, MOGAD can be differentiated from MS with excellent performance (93\% accuracy). The classification model discriminating MOGAD, AQP4+ NMOSD and MS might be useful when antibody assays are not (yet) available. More importantly, our findings may offer clues as to which patients should be tested for the presence of antibodies, particularly in patients without brain lesions. ${ }^{5}$

Several limitations apply to this work. First, this study is a preliminary cross-sectional study with a relatively small sample size and multiple comparisons of brain measures; therefore, further longitudinal studies with larger sample sizes are warranted to confirm our results and detect dynamic MOGAD related changes over time. Second, we used structural MRI measures, including mainly global brain atrophy and WM diffusion measurements. Future research using more sophisticated techniques (eg, TBSS or brain networks) at the regional and voxel level together with functional MRI measures may help reveal a more comprehensive picture of the MOGADspecific brain damage pattern. Additionally, more sensitive sequences (eg, double inversion recovery and phase sensitive inversion recovery) should be used to detect and evaluate (juxta) cortical lesions. Third, this study focused on brain MRI measurements, while spinal cord and optic nerve lesions were not evaluated in this study. Again, a more comprehensive assessment of the whole central nervous system may yield better discrimination. Last, the patients were enrolled after being free of relapses for more than 4 weeks. ${ }^{334}$ Four weeks might not have been long enough to avoid seeing prolonged effects of an acute event.

\section{CONCLUSION}

Patients with early MOGAD have distinct lesion characteristics and brain structural alteration patterns, including lesions predominantly located in the cortical/juxtacortical area and cortical and subcortical GM atrophy without severe WM atrophy and rarefaction, which is different from AQP4+ NMOSD and MS. The subcortical GM volume correlated with clinical disability in MOGAD, implying it is a potential imaging marker for monitoring the disease progression and its use in clinical trials of MOGAD. Finally, our classification model based on structural MRI could help differentiate MOGAD from AQP4+ NMOSD and MS, even in those with no lesions on brain MRI, which could help clinicians in their diagnostic and treatment decisions.

\section{Author affiliations}

'Department of Radiology, Beijing Tiantan Hospital, Capital Medical University, Beijing, China

${ }^{2}$ Tiantan Image Research Center, China National Clinical Research Center for Neurological Diseases, Beijing, China

${ }^{3}$ Department of Radiology, Huashan Hospital Fudan University, Shanghai, China ${ }^{4}$ Institute of Functional and Molecular Medical Imaging, Fudan University, Shanghai, China

${ }^{5}$ Center for Neurology, Beijing Tiantan Hospital, Capital Medical University, Beijing, China 
${ }^{6}$ Translational Medicine Center, China National Clinical Research Center for Neurological Diseases, Beijing, China

${ }^{7}$ Advanced Innovation Center for Human Brain Protection, Capital Medical University, Beijing, China

${ }^{8}$ Department of Radiology, Beijing Bo'ai Hospital, China Rehabilitation Research Center, Beijing, China

${ }^{9}$ Department of Neurology and Tianjin Neurological Institute, Tianjin Medical University General Hospital, Tianjin, China

${ }^{10}$ Department of Radiology and Nuclear Medicine, Neuroscience Campus Amsterdam, VU University Medical Center, Amsterdam, The Netherlands

${ }^{11}$ Queen Square Institute of Neurology and Center for Medical Image Computing, University College London, London, UK

Contributors YD was responsible for the study design, literature research, data acquisition, statistical analysis and manuscript drafting. ZZ was responsible for literature research, data processing, statistical analysis and manuscript drafting. $\mathrm{HL}$ was responsible for data acquisition and manuscript editing. D-CT was responsible for clinical data acquisition. YL was responsible for data acquisition. LY was responsible for data acquisition. CG was responsible for clinical data acquisition. TZ was responsible for literature research and manuscript editing. XZ was responsible for clinical data acquisition. F-DS was responsible for manuscript review. FB was responsible for manuscript review. YL was responsible for guaranty of integrity of the entire study, study design, literature research, statistical analysis, manuscript editing and final approval of this manuscript.

Funding This work was supported by the National Science Foundation of China (Nos. 81870958 and 81571631), the Beijing Municipal Natural Science Foundation for Distinguished Young Scholars (No. JQ20035), the Special Fund of the Paediatric Medical Coordinated Development Centre of Beijing Hospitals Authority (No. XTYB201831) and the Shanghai new star of Medical Court (2018, HL). FB is supported by the NIHR biomedical research centre at UCLH.

Competing interests FB acts as a consultant for Bayer-Schering, Biogen-Idec, GeNeuro, Ixico, Merck-Serono, Novartis and Roche. He has received grants, or grants are pending, from the Amyloid Imaging to Prevent Alzheimer's Disease (AMYPAD) initiative, the Biomedical Research Centre at University College London Hospitals, the Dutch MS Society, ECTRIMS-MAGNIMS, EU-H2020, the Dutch Research Council (NWO), the UK MS Society and the National Institute for Health Research, University College London. He has received payments for the development of educational presentations from Ixico and to his institution from Biogen-Idec and Merck. He is on the editorial board of Radiology, Brain, European Radiology, Multiple Sclerosis Journal and Neurology.

Patient consent for publication Not required.

Provenance and peer review Not commissioned; externally peer reviewed.

Data availability statement Data can be made available on reasonable request by a qualified researcher.

Supplemental material This content has been supplied by the author(s). It has not been vetted by BMJ Publishing Group Limited (BMJ) and may not have been peer-reviewed. Any opinions or recommendations discussed are solely those of the author(s) and are not endorsed by BMJ. BMJ disclaims all liability and responsibility arising from any reliance placed on the content. Where the content includes any translated material, BMJ does not warrant the accuracy and reliability of the translations (including but not limited to local regulations, clinical guidelines, terminology, drug names and drug dosages), and is not responsible for any error and/or omissions arising from translation and adaptation or otherwise.

Open access This is an open access article distributed in accordance with the Creative Commons Attribution Non Commercial (CC BY-NC 4.0) license, which permits others to distribute, remix, adapt, build upon this work non-commercially, and license their derivative works on different terms, provided the original work is properly cited, appropriate credit is given, any changes made indicated, and the use is non-commercial. See: http://creativecommons.org/licenses/by-nc/4.0/.

ORCID iDs

De-Cai Tian http://orcid.org/0000-0002-5153-2491

Liqin Yang http://orcid.org/0000-0003-4383-3574

Fu-Dong Shi http://orcid.org/0000-0002-9675-4637

Yaou Liu http://orcid.org/0000-0002-8753-0260

\section{REFERENCES}

1 Reindl M, Waters P. Myelin oligodendrocyte glycoprotein antibodies in neurological disease. Nat Rev Neurol 2019;15:89-102.

2 Wynford-Thomas R, Jacob A, Tomassini V. Neurological update: MOG antibody disease. J Neurol 2019;266:1280-6.

3 Dubey D, Pittock SJ, Krecke KN, et al. Clinical, radiologic, and prognostic features of myelitis associated with myelin oligodendrocyte glycoprotein autoantibody. JAMA Neurol 2019;76:301-9.
4 Jarius S, Ruprecht K, Kleiter I, et al. MOG-IgG in NMO and related disorders: a multicenter study of 50 patients. Part 2: epidemiology, clinical presentation, radiological and laboratory features, treatment responses, and long-term outcome. $J$ Neuroinflammation 2016:13:280.

5 Jurynczyk M, Geraldes R, Probert F, et al. Distinct brain imaging characteristics of autoantibody-mediated CNS conditions and multiple sclerosis. Brain 2017;140:617-27.

6 Chang VTW, Chang H-M. Review: recent advances in the understanding of the pathophysiology of neuromyelitis optica spectrum disorder. Neuropathol App/ Neurobiol 2020;46:199-218.

7 Haider L, Zrzavy T, Hametner S, et al. The topograpy of demyelination and neurodegeneration in the multiple sclerosis brain. Brain 2016;139:807-15.

8 Wingerchuk DM, Lennon VA, Pittock SJ, et al. Revised diagnostic criteria fo neuromyelitis optica. Neurology 2006;66:1485-9.

9 Brownlee WJ, Use BWJ. Use (and misuse) of the McDonald criteria to diagnose multiple sclerosis. Eur J Neurol 2018;25:209-10.

10 Wakana S, Jiang H, Nagae-Poetscher LM, et al. Fiber tract-based atlas of human white matter anatomy. Radiology 2004;230:77-87.

11 Matthews L, Marasco R, Jenkinson M, et al. Distinction of seropositive NMO spectrum disorder and MS brain lesion distribution. Neurology 2013;80:1330-7.

12 Cobo-Calvo A, Ruiz A, Maillart E, et al. Clinical spectrum and prognostic value of CNS MOG autoimmunity in adults: the MOGADOR study. Neurology 2018;90:e1858-69.

13 Sato DK, Callegaro D, Lana-Peixoto MA, et al. Distinction between MOG antibodypositive and AQP4 antibody-positive NMO spectrum disorders. Neurology 2014;82:474-81.

14 Ramanathan S, Mohammad S, Tantsis E, et al. Clinical course, therapeutic responses and outcomes in relapsing MOG antibody-associated demyelination. J Neurol Neurosurg Psychiatry 2018:89:127-37.

15 Baumann M, Sahin K, Lechner C, et al. Clinical and neuroradiological differences of paediatric acute disseminating encephalomyelitis with and without antibodies to the myelin oligodendrocyte glycoprotein. J Neurol Neurosurg Psychiatry 2015;86:265-72.

16 Kim S-M, Woodhall MR, Kim J-S, et al. Antibodies to MOG in adults with inflammatory demyelinating disease of the CNS. Neurol Neuroimmunol Neuroinflamm 2015;2:e163.

17 Höftberger R, Sepulveda M, Armangue T, et al. Antibodies to MOG and AQP4 in adults with neuromyelitis optica and suspected limited forms of the disease. Mult Scler 2015;21:866-74

18 Chen C, Liu C, Fang L, et al. Different magnetic resonance imaging features between MOG antibody- and AQP4 antibody-mediated disease: a Chinese cohort study. J Neurol Sci 2019:405:116430.

19 Ogawa R, Nakashima I, Takahashi T, et al. Mog antibody-positive, benign, unilateral, cerebral cortical encephalitis with epilepsy. Neurol Neuroimmunol Neuroinflamm 2017:4:e322

20 Fujimori J, Takai Y, Nakashima I, et al. Bilateral frontal cortex encephalitis and paraparesis in a patient with anti-MOG antibodies. J Neurol Neurosurg Psychiatry 2017;88:534-6.

21 Hamid SHM, Whittam D, Saviour M, et al. Seizures and encephalitis in myelin oligodendrocyte glycoprotein lgG disease vs aquaporin $4 \mathrm{lg}$ G disease. JAMA Neurol 2018;75:65.

22 Salama S, Khan M, Shanechi A, et al. MRI differences between MOG antibody disease and AQP4 NMOSD. Mult Scler 2020:26:1854-65.

23 Hyun J-W, Huh S-Y, Shin H-J, et al. Evaluation of brain lesion distribution criteria at disease onset in differentiating MS from NMOSD and MOG-IgG-associated encephalomyelitis. Mult Scler 2019;25:585-90.

24 Friese MA, Schattling B, Fugger L. Mechanisms of neurodegeneration and axonal dysfunction in multiple sclerosis. Nat Rev Neurol 2014;10:225-38.

25 Spadaro M, Gerdes LA, Mayer MC, et al. Histopathology and clinical course of MOGantibody-associated encephalomyelitis. Ann Clin Trans/ Neurol 2015;2:295-301.

26 Wang JJ, Jaunmuktane Z, Mummery C, et al. Inflammatory demyelination without astrocyte loss in MOG antibody-positive NMOSD. Neurology 2016:87:229-31.

27 Sun J, Zhang N, Wang Q, et al. Normal-appearing cerebellar damage in neuromyelitis optica spectrum disorder. AJNR Am J Neuroradiol 2019;40:1156-61.

28 Eshaghi A, Marinescu RV, Young AL, et al. Progression of regional grey matter atrophy in multiple sclerosis. Brain 2018:141:1665-77.

29 Rossi A, Crane JM, Verkman AS. Aquaporin-4 MZ isoform: brain expression supramolecular assembly and neuromyelitis optica antibody binding. Glia 2011:59:1056-63.

30 Suzuki M, Obara K, Sasaki Y, et al. Comparison of perivascular astrocytic structure between white matter and gray matter of rats. Brain Res 2003:992:294-7.

31 Liu Y, Wang J, Daams M, et al. Differential patterns of spinal cord and brain atrophy in NMO and MS. Neurology 2015;84:1465-72.

32 Höftberger R, Guo Y, Flanagan EP, et al. The pathology of central nervous system inflammatory demyelinating disease accompanying myelin oligodendrocyte glycoprotein autoantibody. Acta Neuropathol 2020;139:875-92.

33 Cotton F, Weiner HL, Jolesz FA, et al. MRI contrast uptake in new lesions in relapsingremitting MS followed at Weekly intervals. Neurology 2003:60:640-6.

34 Jenkins TM, Ciccarelli O, Atzori M, et al. Early pericalcarine atrophy in acute optic neuritis is associated with conversion to multiple sclerosis. J Neurol Neurosurg Psychiatry 2011;82:1017-21. 